See discussions, stats, and author profiles for this publication at: https://www.researchgate.net/publication/336002264

\title{
Thermal-Conductivity Enhancement by Surface Electromagnetic Waves Propagating along Multilayered Structures with Asymmetric Surrounding Media
}

Article in Physical Review Applied · September 2019

DOI: 10.1103/PhysRevApplied.12.034044

CITATIONS

READS

0

4

5 authors, including:

Jose Ordonez-Miranda

CNRS

108 PUBLICATIONS 844 CITATIONS

SEE PROFILE

Some of the authors of this publication are also working on these related projects:

Composites Thermal Conductivity View project

Effective interface thermal resistance and thermal conductivity of dielectric nanolayers View project 


\title{
Thermal-Conductivity Enhancement by Surface Electromagnetic Waves Propagating along Multilayered Structures with Asymmetric Surrounding Media
}

\author{
Mikyung Lim, ${ }^{1}$ Jose Ordonez-Miranda, ${ }^{2}$ Seung S. Lee, ${ }^{1}$ Bong Jae Lee,,${ }^{1,}$ and Sebastian Volz ${ }^{3,4, \dagger}$ \\ ${ }^{1}$ Department of Mechanical Engineering, Korea Advanced Institute of Science and Technology, Daejeon 34141, \\ South Korea \\ ${ }^{2}$ Institut Pprime, CNRS, Université de Poitiers, ISAE-ENSMA, F-86962 Futuroscope Chasseneuil, France \\ ${ }^{3}$ LIMMS/CNRS-IIS(UMI2820), Institute of Industrial Science, University of Tokyo, Tokyo 153-8505, Japan \\ ${ }^{4}$ Laboratoire d'Energétique Moléculaire et Macroscopique, Combustion, UPR CNRS 288 CentraleSupélec, \\ Université Paris-Saclay, France
}

(Received 1 June 2019; revised manuscript received 14 July 2019; published 23 September 2019)

\begin{abstract}
The enhancement of thermal conductivity via surface electromagnetic waves (SEWs) supported in nanostructures has recently attracted attention as a remedy for issues raised due to the reduction of thermal conductivity in nanoscale confinement. Among them, multilayered structures on a substrate are prevalent in nanosized systems, such as electronic nanodevices, meaning that analysis on those structures is indispensable. In this work, three basic multilayered structures are selected and the analytical expressions for SEWs supported in each structure are derived. This analytical approach enables us to figure out which factors are crucial for enhancing SEW thermal conductivity using multilayers. It is also found that the solution can be extended to various materials and provides guidelines on which configurations are desirable for increasing the thermal conductivity. Furthermore, the analytical solutions reduce the calculation time significantly such that the optimal configuration, which can yield a SEW thermal conductivity of $1.27 \mathrm{~W} / \mathrm{m} \mathrm{K}$, corresponding to $90 \%$ of the thermal conductivity of bulk glass, is found using a genetic algorithm. This study thus provides a method for efficiently managing thermal issues in nanosized devices.
\end{abstract}

DOI: 10.1103/PhysRevApplied.12.034044

\section{INTRODUCTION}

Thermal-energy transport via surface electromagnetic waves (SEWs) propagating along thin films has been intensively investigated over the past decade [1-6] because of its potential application to compensation of the reduction of the thermal performance of systems when their sizes are reduced to the nanoscale [2]. In particular, the booming miniaturization of electronic devices has been leading to a decrease in the size of their components to a few nanometers, which is smaller than the mean free path of the electrons or phonons of the constituting materials and reduces their effective thermal conductivities [7]. Given that the problem of heat spreading can generate hot spots, which overheat the electronic nanodevices and deteriorate their performance [8], the additional energy-transport mechanism driven by SEWs supported in nanosized systems has been regarded as a promising remedy to tackle this issue of overheating [1-6].

SEWs are bounded at the interfaces along which they propagate, carrying energy [9-12]. The propagation length

\footnotetext{
*bongjae.lee@kaist.ac.kr

†volz@iis.u-tokyo.ac.jp
}

of these SEWs can be drastically increased as the thickness of the suspended glass layer decreases [1,3]; however, when this single glass layer is placed on a substrate (asymmetric surrounding media), the frequency interval over which SEWs can be supported becomes severely restricted [3] and, thus, additional energy transport via SEWs is rather limited. Furthermore, besides the single glass layer, thermal-conductivity enhancement by SEWs within multilayered structures should also be considered for a wide range of applications.

Although SEWs supported in periodic multilayered structures have been widely investigated [13-19], their contribution in the field of thermal conductivity has rarely been reported. A previous work [4] has explored the thermal-conductivity enhancement of a suspended nanolayered system due to the propagation of SEWs by regarding nanolayers as a single layer with an effective permittivity. Because this effective permittivity only depends on the relative thickness of the constituting layers, it cannot fully take account of the configuration of the nanolayers. Accordingly, this means cannot be safely extended when a structure consisting of a few layers on a substrate, which is a more practical configuration than the suspended periodic multilayered structure, is considered. 
In this work, various structures that consist of a few layers on a substrate are evaluated and the best structure that can have the largest SEW thermal conductivity is discussed. The exact propagation length of the SEWs supported in each configuration is obtained numerically first and the approximate analytical solution is derived for each configuration. Then, based on this analytical description, the effects of the constituting layers and the configurations on the propagation lengths of the SEWs are thoroughly explored to achieve a significant enhancement in SEW thermal conductivity. Finally, the configuration is optimized using a genetic algorithm to maximize the SEW thermal conductivity by employing the analytical solutions.

Depending on their nature, thermally excited SEWs have been classified into the following modes: surface phonon (plasmon) polaritons, Zenneck modes, and transverse-magnetic (TM) guided modes $[11,20]$. Among these modes, the focus is usually placed on surface polaritons [9] because they can significantly enhance the tunneling of evanescent waves across a vacuum gap, their dispersion relation can be tailored by the structure, and thus they can be employed in controlling near-field thermal radiation [21-23], near-field thermophotovoltaics [15,24], and microscopy [25]. However, a recent experimental study [20] has shown that the Zenneck modes can provide longer propagation lengths in wider frequency intervals than surface polaritons, which means that Zenneck modes are more beneficial in carrying energy than these latter polaritons. Throughout this paper, both Zenneck modes and surface polaritons will be analyzed, while TM guided modes will be not considered, because they are not coupled to the thermal sources.

\section{THERMAL-CONDUCTIVITY MODELING AND DISPERSION RELATION}

The effective SEW thermal conductivity, $k_{\mathrm{SEW}}$, of layered structures due to the propagation of SEWs is given by the following $[1,3]$ :

$$
k_{\mathrm{SEW}}=\frac{1}{4 \pi d} \int_{0}^{\infty} \hbar \omega \Lambda \beta_{R} \frac{\partial f_{0}}{\partial T} d \omega=\int_{0}^{\infty} k_{\omega} d \omega,
$$

where $\hbar$ is the Planck constant divided by $2 \pi, \omega$ is the angular frequency, $d$ is the total thickness of the multilayer with average temperature $T$, and $f_{0}$ is the BoseEinstein distribution function. The values of the unknown parameters $\beta_{R}=\operatorname{Re}(\beta)$ and the propagation length $\Lambda=$ $1 /[2 \operatorname{Im}(\beta)]$ can be obtained, respectively, from the real and imaginary parts of the in-plane wave vector $\beta=$ $\beta_{R}+i \beta_{I}$, which is defined by the dispersion relation of the SEWs propagating in a given layered structure. Considering that $\Lambda$ cannot be longer than the system size $L$, throughout the paper the thermal conductivity in Eq. (1) is then calculated using $\Lambda_{\text {eff }}$, which is defined as $1 / \Lambda_{\text {eff }}=$ $1 / \Lambda+1 / L$. The frequency interval for integration is taken as that for Zenneck modes and surface phonon polaritons [20]. In Eq. (1), the spectral thermal conductivity, $k_{\omega}$, is proportional to the product of $\hbar \omega\left(\partial f_{0} / \partial T\right)$ and $\Lambda \beta_{R}$. Given that the value of $\hbar \omega\left(\partial f_{0} / \partial T\right)$ is larger at lower frequencies, the structure should support SEWs with large propagation lengths at low frequency such that they have large thermal conductivities.

\section{A. Glass-dielectric structures}

Let us first consider the glass-dielectric (GD) structure [see Fig. 1(a)], which supports the propagation of the SEWs with the following dispersion relation $[4,26]$ :

$$
\frac{\tanh \left(p_{d} d_{d}\right)+\alpha_{s d}}{1+\alpha_{s d} \tanh \left(p_{d} d_{d}\right)}=-\alpha_{g d} \frac{\tanh \left(p_{g} d_{g}\right)+\alpha_{0 g}}{1+\alpha_{0 g} \tanh \left(p_{g} d_{g}\right)},
$$

where $\alpha_{i j}=\epsilon_{i} p_{j} / \epsilon_{j} p_{i}$. Here, $\epsilon_{i}$ refers to the dielectric function of each layer and the transverse wave vector $p_{i}$ is given by $p_{i}^{2}=\beta^{2}-\epsilon_{i} k_{0}^{2}$, with $k_{0}=\omega / c_{0}$ being the wave vector in a vacuum. The subscripts $0, g, d$, and $s$ denote vacuum, glass, dielectric, and substrate, respectively, and $c_{0}$ is the speed of light in a vacuum. The solution of Eq. (2) (a) GD
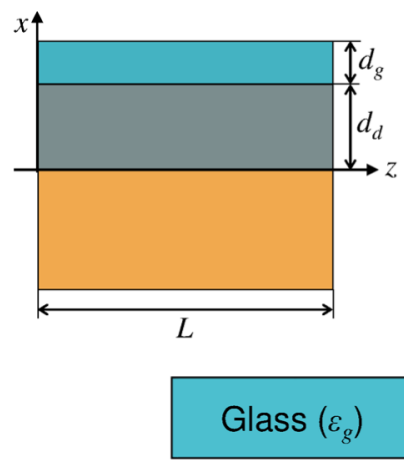

(b) $D G$
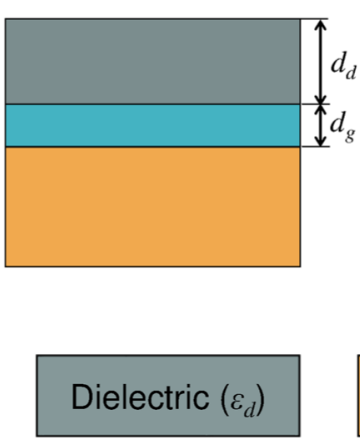

(c) GDG

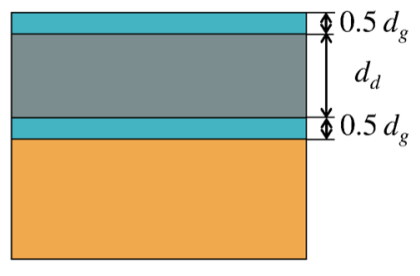

Substrate $\left(\varepsilon_{s}\right)$
FIG. 1. The schematics of the layered structures that are considered in this work: (a) glass-dielectric (GD), dielectric-glass (DG), and (c) glass-dielectric-glass (GDG), deposited on a semi-infinite substrate. The volume-filling factor of the glass is kept constant for the three structures. Subscripts $g, d$, and $s$ denote glass, dielectric, and substrate, respectively, where $\epsilon_{n}$ is the dielectric function of layer $n$. 

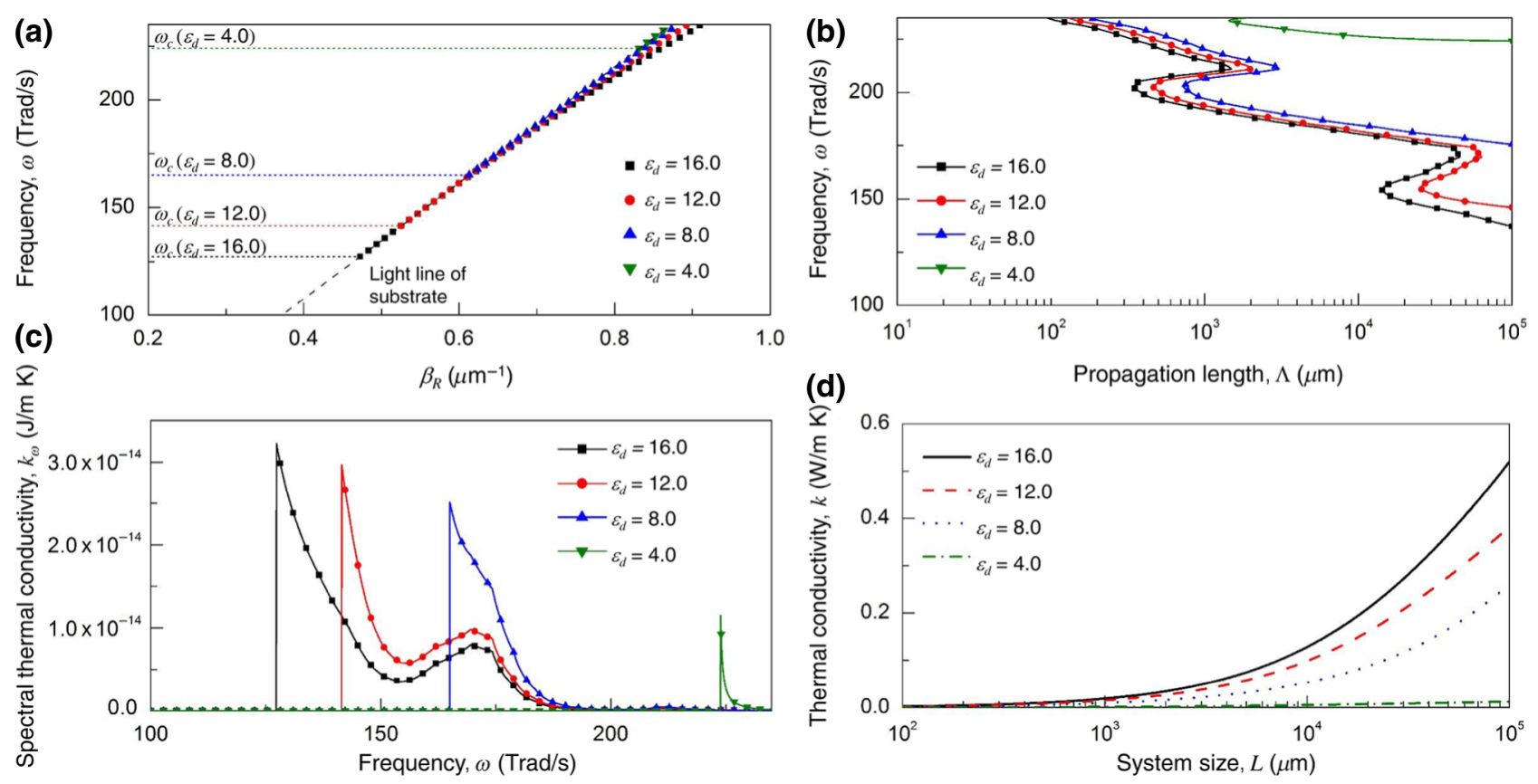

FIG. 2. The dispersion relation and SEW thermal conductivity of the GD structure with dielectric layers of various permittivities. The thicknesses of the glass and dielectric layers are set to $20 \mathrm{~nm}$ and $680 \mathrm{~nm}$, respectively (total thickness $d=700 \mathrm{~nm}$ ), and the average temperature $T$ is set to $300 \mathrm{~K}$. The frequency-dependent (a) $\beta_{R}$, (b) propagation length, and (c) spectral thermal conductivity when the system size is set to $10 \mathrm{~cm}$. (d) The resulting thermal conductivity, depending on the size of the system.

for the complex wave vector $\beta$ is obtained numerically by the secant method with a proper choice of the initial point and using the glass dielectric function reported in Ref. [27]. The dielectric function of the substrate is assumed to be $\epsilon_{s}=1.24$ throughout this work, as done in Refs. [3] and [4].

The wave vector $\beta_{R}$ and the propagation lengths of the SEWs are shown in Figs. 2(a) and 2(b), for different values of the dielectric function $\epsilon_{d}$. Note that $\beta_{R}$ is close to the light line of the substrate (i.e., $\beta_{R} \approx \sqrt{\epsilon_{s}} k_{0}$ ) except for the high frequencies $(\omega>200 \mathrm{Trad} / \mathrm{s})$. In this work, only this photonlike mode is considered, because it has much larger propagation lengths than the phononlike mode, which is found to have propagation length smaller than $1 \mu \mathrm{m}$, as in Ref. [1]. It can be seen that a frequency interval in which the solution exists varies with $\epsilon_{d}$. We set the lowest value of the frequency at which the solution can exist as a cutoff frequency, $\omega_{c}$, for each case. The estimation of $\omega_{c}$ for different configurations is crucial because at $\omega_{c}$, SEWs with the longest propagation length can be supported in the structures, as can be seen in Fig. 2(b). With the system size taken to be $10 \mathrm{~cm}$, the corresponding spectral thermal conductivity, $k_{\omega}$, for each case is plotted in Fig. 2(c), which shows that $k_{\omega}$ reaches its highest value at $\omega_{c}$ for each case. Although the propagation length is smaller for higher values of $\epsilon_{d}$ at the same frequency, the wave vector can be extended to lower frequencies with higher values of $\epsilon_{d}$ [see Fig. 2(b)]. As a result, in Fig. 2(d), when $\epsilon_{d}=16.0$ and the system size is set to $10 \mathrm{~cm}$, the corresponding thermal conductivity is $0.52 \mathrm{~W} / \mathrm{m} \mathrm{K}$, which is $37 \%$ of the thermal conductivity of bulk glass. Although the solutions shown in Fig. 2 can be obtained numerically, it is hard to see which factors affect the propagation length or the frequency interval. Alternatively, an analytical solution, which may provide a physical insight into which structures are beneficial for enhancing SEW heat transfer, is derived with several approximations.

Given that the propagation lengths of SEWs decrease as the thickness of the glass layer increases [1,3], the case of glass with a very small thickness will be considered to derive an analytical solution of Eq. (2). Under this thin-film approximation (i.e., $\left.\left|p_{g}\right| d_{g} \ll 1\right)$ and up to a first order on $\left|p_{g}\right| d_{g} \ll 1$, Eq. (2) can be expressed as follows:

$$
\frac{A+\left(\epsilon_{s} p_{d} / \epsilon_{d} p_{s}\right)}{1+A\left(\epsilon_{s} p_{d} / \epsilon_{d} p_{s}\right)}=-\frac{\epsilon_{g} p_{d}}{\epsilon_{d} p_{g}} \frac{p_{g} d_{g}+\left(\epsilon_{0} p_{g} / \epsilon_{g} p_{0}\right)}{1+\left(\epsilon_{0} p_{g} / \epsilon_{g} p_{0}\right) p_{g} d_{g}},
$$

where $A=\tanh \left(p_{d} d_{d}\right)$. The solution of Eq. (3) for $p_{s}$ can be expressed as follows:

$$
p_{s}=-\frac{\epsilon_{s} p_{d}\left[A \epsilon_{g}\left(\epsilon_{0}+d_{g} \epsilon_{g} p_{0}\right) p_{d}+\epsilon_{d}\left(\epsilon_{g} p_{0}+d_{g} \epsilon_{0} p_{g}^{2}\right)\right]}{\epsilon_{d}\left[\epsilon_{g}\left(\epsilon_{0}+d_{g} \epsilon_{g} p_{0}\right) p_{d}+A \epsilon_{d}\left(\epsilon_{g} p_{0}+d_{g} \epsilon_{0} p_{g}^{2}\right)\right]} .
$$

Considering that $p_{s} \rightarrow 0$ when $\beta_{R} \rightarrow \sqrt{\epsilon_{s}} k_{0}$ and $\beta_{I} \rightarrow 0$, $p_{i}^{2}=p_{s}^{2}+\left(\epsilon_{s}-\epsilon_{i}\right) k_{0}^{2}$ can be approximated as $p_{i}^{2} \approx\left(\epsilon_{s}-\right.$ 
$\left.\epsilon_{i}\right) k_{0}^{2}$. Taking into account the condition $\operatorname{Re}\left(p_{i}\right)>0$ to ensure the bounding of the SEW to the interfaces, $p_{i} \approx$ $\sqrt{\epsilon_{s}-\epsilon_{i}} k_{0}=D_{i} k_{0}$. The application of $p_{i} \approx D_{i} k_{0}$ to Eq. (4) provides the approximate solution as follows:

$$
p_{s}=-\frac{\epsilon_{s} D_{d} k_{0}\left[A^{\prime} D_{d} \epsilon_{g}\left(\epsilon_{0}+D_{0} d_{g} \epsilon_{g} k_{0}\right)+\epsilon_{d}\left(D_{0} \epsilon_{g}+d_{g} D_{g}^{2} \epsilon_{0} k_{0}\right)\right]}{\epsilon_{d}\left[D_{d} \epsilon_{g}\left(\epsilon_{0}+D_{0} d_{g} \epsilon_{g} k_{0}\right)+A^{\prime} \epsilon_{d}\left(D_{0} \epsilon_{g}+d_{g} D_{g}^{2} \epsilon_{0} k_{0}\right)\right]},
$$

where $A^{\prime}=\tanh \left(D_{d} k_{0} d_{d}\right)$. Figures 3(a) and 3(b) show the analytical solution for $\beta_{R}$ and the propagation length, obtained using the relation $\beta^{2}=p_{s}^{2}+\epsilon_{s} k_{0}^{2}$. Although several approximations are made, it is readily seen that the approximate solution agrees reasonably with the numerical one. In order to analyze the propagation length and the frequency interval in which the SEWs can exist, the analytical solution for $\beta_{I}$ is also derived. From the relation $\beta^{2}=p_{s}^{2}+\epsilon_{s} k_{0}^{2}$, when $\beta_{R} \rightarrow \sqrt{\epsilon_{s}} k_{0}, \beta_{I}$ can be approximated as $\left(p_{s, R} p_{s, I}\right) / \sqrt{\epsilon_{s}} k_{0}$ [3]. Here, $p_{s, R}$ and $p_{s, I}$ are the real and imaginary parts of $p_{s}$, respectively. Note that $D_{0}$ is purely real and $D_{d}$ is purely imaginary, such that $A^{\prime}=$ $\tanh \left(D_{d} k_{0} d_{d}\right)$ can be expressed as $A^{\prime}=i \tan \left(D_{d, I} k_{0} d_{d}\right)=$ $i A_{I}^{\prime}$ with $D_{d}=i D_{d, I}$. Equation (5) can then be rewritten as follows:

$$
p_{s}=-\frac{\epsilon_{s} D_{d, I} k_{0}\left\{-A_{I}^{\prime} D_{d, I} \epsilon_{g}\left(\epsilon_{0}+D_{0} d_{g} \epsilon_{g} k_{0}\right)+\epsilon_{d}\left[D_{0} \epsilon_{g}+d_{g}\left(\epsilon_{s}-\epsilon_{g}\right) \epsilon_{0} k_{0}\right]\right\}}{\epsilon_{d}\left\{D_{d, I} \epsilon_{g}\left(\epsilon_{0}+D_{0} d_{g} \epsilon_{g} k_{0}\right)+A_{I}^{\prime} \epsilon_{d}\left[D_{0} \epsilon_{g}+d_{g}\left(\epsilon_{s}-\epsilon_{g}\right) \epsilon_{0} k_{0}\right]\right\}}
$$

where $\epsilon_{g}=\epsilon_{g, R}+i \epsilon_{g, I}$. After conducting the complex expansion of Eq. (6), $p_{s, R}$ and $p_{s, I}$ can be obtained. Using the relation $\beta_{I} \approx\left(p_{s, R} p_{s, I}\right) / \sqrt{\epsilon_{s}} k_{0}$ and assuming that $p_{g} d_{g}$ is small, the approximate explicit expression for $\beta_{I}$ can be obtained as follows:

$$
\beta_{I, \mathrm{GD}} \approx \frac{D_{d, I}^{3} d_{g} \epsilon_{g, I} \epsilon_{s}^{1.5}\left(1+A_{I}^{\prime 2}\right)\left(A_{I}^{\prime} D_{d, I} \epsilon_{0}-D_{0} \epsilon_{d}\right)\left[D_{0}^{2}\left(\epsilon_{g, R}^{2}+\epsilon_{g, I}^{2}\right)+\epsilon_{0}^{2} \epsilon_{s}\right] k_{0}^{2}}{\epsilon_{d}\left(D_{d, I} \epsilon_{0}+A_{I}^{\prime} D_{0} \epsilon_{d}\right)^{3}\left(\epsilon_{g, R}^{2}+\epsilon_{g, I}^{2}\right)} .
$$

The predictions of Eq. (7) for $\beta_{I, \mathrm{GD}}$ are shown in Fig.3(c) in comparison with the numerical solution obtained from Eq. (2). Although Eq. (7) is derived through different assumptions, a negligible discrepancy between the analytical and numerical solutions is observed except for the higher-frequency regime, where the spectral thermal conductivity has smaller values. Taking into account that $D_{d, I}>0, d_{g}>0, \epsilon_{s}>0, \epsilon_{d}>0$, and $\epsilon_{g, I}>0$, $\beta_{I, \mathrm{GD}}$ is positive only when $\left(A_{I}^{\prime} D_{d, I} \epsilon_{0}-D_{0} \epsilon_{d}\right) /\left(D_{d, I} \epsilon_{0}+\right.$ $\left.A_{I}^{\prime} D_{0} \epsilon_{d}\right)>0$. In other words, SEWs propagating along the GD structure exist for frequencies satisfying this latter condition, which varies with $\epsilon_{d}$, as can be seen in Fig. 3(c). Higher values of $\epsilon_{d}$ satisfy $\left(A_{I}^{\prime} D_{d, I} \epsilon_{0}-D_{0} \epsilon_{d}\right) /\left(D_{d, I} \epsilon_{0}+\right.$ $\left.A_{I}^{\prime} D_{0} \epsilon_{d}\right)>0$ at lower frequencies, such that the GD structure with higher $\epsilon_{d}$ can have a higher SEW thermal conductivity, as shown in Fig. 2(d).
Let us see how the other factors affect the propagation length. If we change $d_{g}$ under the assumption that $p_{g} d_{g}$ is small, as in Fig. 4(a), the propagation length increases as $d_{g}$ decreases and the frequency interval in which the solution exists is not changed much by changing $d_{g}$. This trend agrees well with the prediction of Eq. (7), as $\beta_{I, \mathrm{GD}}$ is proportional to $d_{g}$ and $\left(A_{I}^{\prime} D_{d, I} \epsilon_{0}-D_{0} \epsilon_{d}\right) /\left(D_{d, I} \epsilon_{0}+\right.$ $\left.A_{I}^{\prime} D_{0} \epsilon_{d}\right)$ is not a function of $d_{g}$. If we recall that $A_{I}^{\prime}=$ $\tan \left(D_{d, I} k_{0} d_{d}\right)$, the frequency interval in which the solution exists increases as $d_{d}$ increases. It should be noted that when $d_{d}=580 \mathrm{~nm}$, the solution in Eq. (7) exists at relatively high frequencies, where the spectral thermal conductivity has small values. It is also worthwhile mentioning that for $d_{d}=380 \mathrm{~nm}$ and $\epsilon_{d}=12.0$, the solution does not exist within the frequency range over which $\mathrm{SiO}_{2}$ can emit. Thus, a proper choice of the dielectric layer 

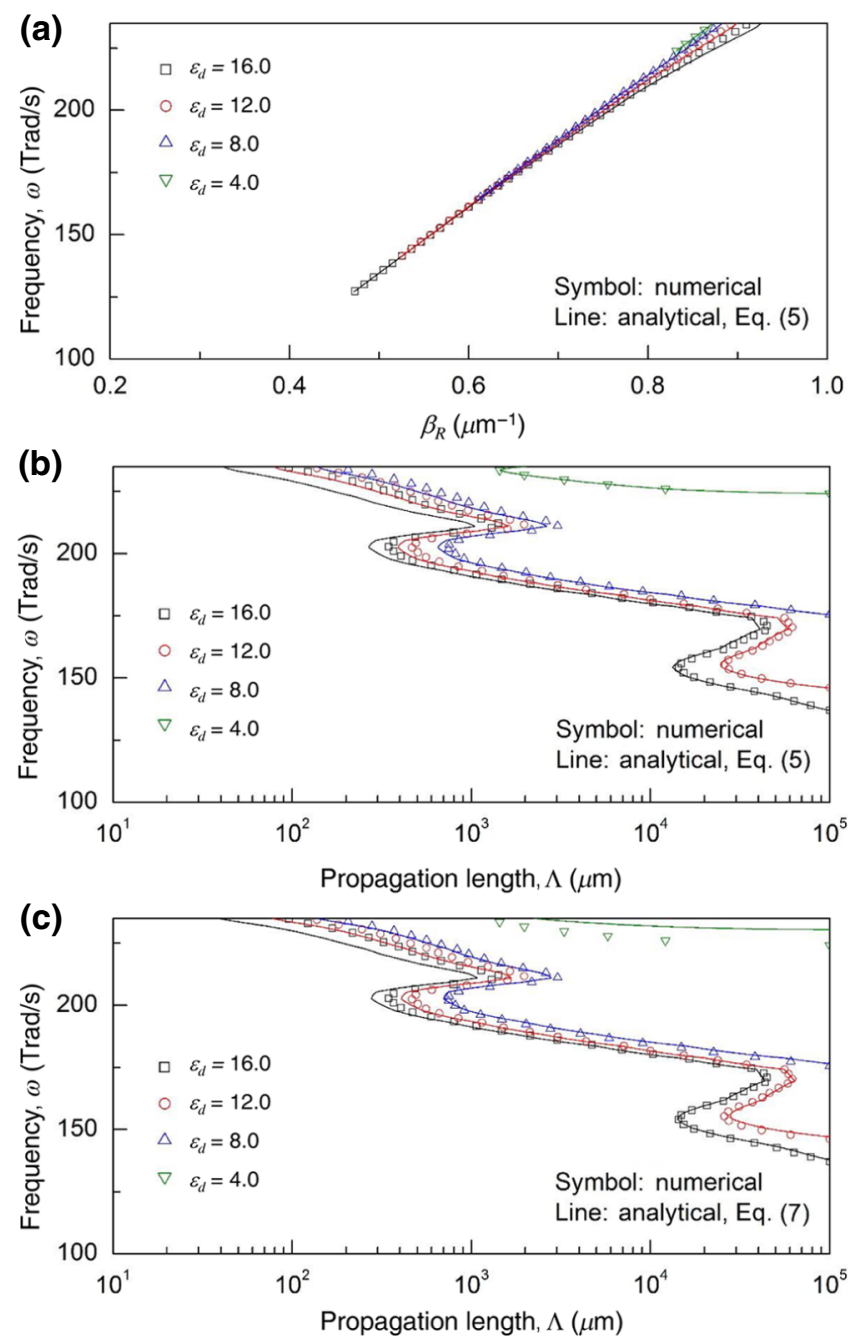

FIG. 3. (a) The dispersion relation and (b),(c) the propagation length of SEWs in the GD structure obtained via a numerical method and an analytical solution. The analytical solution is calculated using Eq.(5) for (a),(b) and Eq.(7) for (c). The conditions are the same as in Fig. 2.

thickness is required to exploit SEWs for the purpose of carrying energy.

Given that the term $\left(A_{I}^{\prime} D_{d, I} \epsilon_{0}-D_{0} \epsilon_{d}\right) /\left(D_{d, I} \epsilon_{0}+A_{I}^{\prime}\right.$ $D_{0} \epsilon_{d}$ ) does not involve the dielectric function of glass, $\epsilon_{g}$, the frequency interval in which the solution exists remains almost invariant under the changes of $\epsilon_{g}$, as can be seen in Figs. 4(b) and 4(c). Although a slight mismatch between the analytical and numerical solutions is found due to large absolute values of the dielectric function of $\mathrm{SiC},\left|\epsilon_{\mathrm{SiC}}\right|$, the frequency interval does not change much, even if the glass is replaced by $\mathrm{SiC}$ or $\mathrm{MgF}_{2}$, in contrast to the notable difference in the frequency interval when $\epsilon_{d}$ is varied from 12.0 to 16.0 [see Figs. 4(b) and 4(c)].

\section{B. Dielectric-glass structure}

In this section, the dielectric-glass (DG) structure introduced in Fig. 1(b) is analyzed to determine its
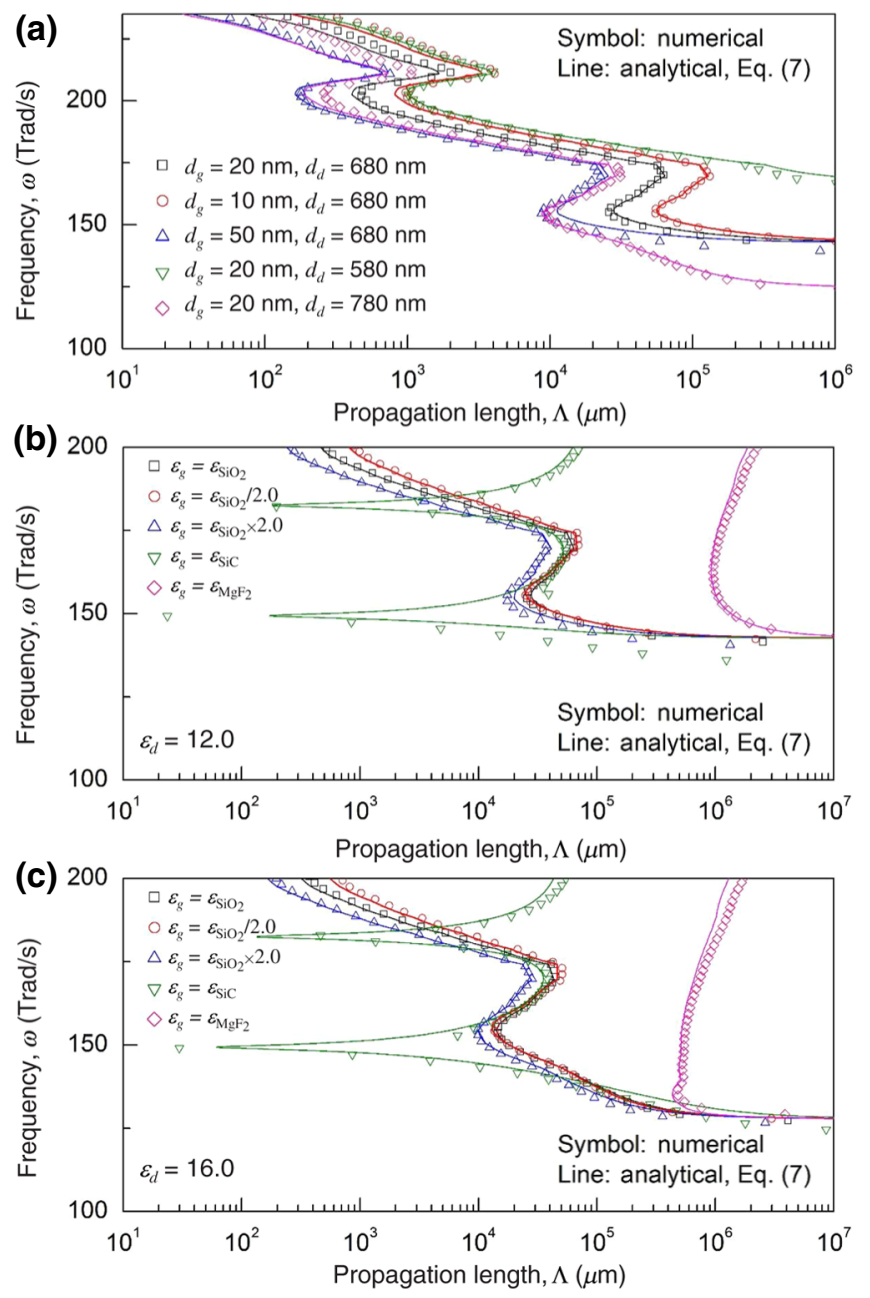

FIG. 4. The propagation lengths of SEWs propagating along the GD structure with different configurations: (a) when the thickness of each layer varies while the dielectric function of the dielectric layer $\epsilon_{d}$ remains at 12.0 ; (b),(c) when the dielectric function of the glass layer varies while the thicknesses of the glass $\left(\mathrm{SiC}\right.$ or $\left.\mathrm{MgF}_{2}\right)$ layer and the dielectric layer are set to $20 \mathrm{~nm}$ and $680 \mathrm{~nm}$, respectively. The dielectric function of the dielectric layer is (b) 12.0 and (c) 16.0 .

performance for carrying thermal energy with SEWs. As in Eq. (2), the dispersion relation for the structure DG can be written as follows $[4,26]$ :

$$
\frac{\tanh \left(p_{g} d_{g}\right)+\alpha_{s g}}{1+\alpha_{s g} \tanh \left(p_{g} d_{g}\right)}=-\alpha_{d g} \frac{\tanh \left(p_{d} d_{d}\right)+\alpha_{0 d}}{1+\alpha_{0 d} \tanh \left(p_{d} d_{d}\right)}
$$

where all the parameters have been previously defined. As in Eqs. (3)-(7), the simplification of Eq. (8) under the assumption of $\left|p_{g}\right| d_{g} \ll 1$ yields the following $\beta_{I}$ for the DG structure:

$$
\beta_{I, \mathrm{DG}} \approx \frac{D_{d, I} d_{g} \epsilon_{g, I} \epsilon_{s}^{1.5}\left(A_{I}^{\prime} D_{d, I} \epsilon_{0}-D_{0} \epsilon_{d}\right) B_{\mathrm{DG}} k_{0}^{2}}{\epsilon_{d}^{3}\left(D_{d, I} \epsilon_{0}+A_{I}^{\prime} D_{0} \epsilon_{d}\right)^{3}\left(\epsilon_{g, R}^{2}+\epsilon_{g, I}^{2}\right)},
$$


where

$$
\begin{aligned}
B_{\mathrm{DG}}= & \epsilon_{d}^{2}\left(\epsilon_{d}-\epsilon_{s}\right)\left[D_{0}^{2}\left(\epsilon_{g, R}^{2}+\epsilon_{g, I}^{2}\right)+\epsilon_{0}^{2} \epsilon_{s}\right] \\
& +A_{I}^{\prime 2}\left[\epsilon_{0}^{2}\left(\epsilon_{g, R}^{2}+\epsilon_{g, I}^{2}\right)\left(\epsilon_{d}-\epsilon_{s}\right)^{2}+D_{0}^{2} \epsilon_{d}^{4} \epsilon_{s}\right] \\
& +2 A_{I}^{\prime} D_{0} D_{d, I} \epsilon_{0} \epsilon_{d}\left[\epsilon_{d}^{2} \epsilon_{s}-\left(\epsilon_{d}-\epsilon_{s}\right)\left(\epsilon_{g, R}^{2}+\epsilon_{g, I}^{2}\right)\right] .
\end{aligned}
$$

The difference between $\beta_{I, \mathrm{DG}}$ and $\beta_{I, \mathrm{GD}}$ can then be expressed as follows:

$$
\beta_{I, \mathrm{DG}}-\beta_{I, \mathrm{GD}}=\frac{D_{d, I} d_{g} \epsilon_{g, I} \epsilon_{s}^{1.5}\left(A_{I}^{\prime} D_{d, I} \epsilon_{0}-D_{0} \epsilon_{d}\right) C k_{0}^{2}}{\epsilon_{d}^{3}\left(D_{d, I} \epsilon_{0}+A_{I}^{\prime} D_{0} \epsilon_{d}\right)^{3}\left(\epsilon_{g, R}^{2}+\epsilon_{g, I}^{2}\right)}
$$

where

$$
\begin{aligned}
C= & A_{I}^{\prime}\left\{2 D_{0} D_{d, I} \epsilon_{0} \epsilon_{d}+A_{I}^{\prime}\left[\epsilon_{d}^{2}\left(\epsilon_{s}-\epsilon_{0}\right)+\epsilon_{0}^{2}\left(\epsilon_{s}-\epsilon_{d}\right)\right]\right\} \\
& \times\left[\epsilon_{d}^{2} \epsilon_{s}-\left(\epsilon_{d}-\epsilon_{s}\right)\left|\epsilon_{g}\right|^{2}\right] .
\end{aligned}
$$

Note that the SEWs propagating along the GD structure only exist when $\left(A_{I}^{\prime} D_{d, I} \epsilon_{0}-D_{0} \epsilon_{d}\right) /\left(D_{d, I} \epsilon_{0}+A_{I}^{\prime} D_{0} \epsilon_{d}\right)>$ 0 . Also, $D_{d, I}>0, d_{g}>0, \epsilon_{s}>0, \epsilon_{d}>0$, and $\epsilon_{g, I}>0$. Accordingly, the sign of $\beta_{I, \mathrm{DG}}-\beta_{I, \mathrm{GD}}$ is the same as the sign of $C$ described in Eq. (12). Thus, $A_{I}^{\prime}\left\{2 D_{0} D_{d, I} \epsilon_{0} \epsilon_{d}-\right.$ $\left.A_{I}^{\prime}\left[\epsilon_{d}^{2}\left(\epsilon_{s}-\epsilon_{0}\right)+\epsilon_{0}^{2}\left(\epsilon_{s}-\epsilon_{d}\right)\right]\right\}$ and $\epsilon_{d}^{2} \epsilon_{s}-\left(\epsilon_{d}-\epsilon_{s}\right)\left|\epsilon_{g}\right|^{2}$ can both act as indicators for determining which structure supports SEWs with longer propagation lengths. In Fig. 5, for the given configuration, $A_{I}^{\prime}\left\{2 D_{0} D_{d, I} \epsilon_{0} \epsilon_{d}-A_{I}^{\prime}\left[\epsilon_{d}^{2}\left(\epsilon_{s}-\right.\right.\right.$ $\left.\left.\left.\epsilon_{0}\right)+\epsilon_{0}^{2}\left(\epsilon_{s}-\epsilon_{d}\right)\right]\right\}$ is found to remain positive in the frequency interval in which the solution exists. Therefore, when $\epsilon_{d}^{2} \epsilon_{s}-\left(\epsilon_{d}-\epsilon_{s}\right)\left|\epsilon_{g}\right|^{2}<0, \beta_{I, \mathrm{DG}}$ has a smaller value than $\beta_{I, \mathrm{GD}}$, meaning that $\Lambda_{\mathrm{DG}}>\Lambda_{\mathrm{GD}}$. Except for this frequency interval, it can be readily noted in Fig. 5 that the GD structure supports SEWs with longer propagation lengths. For this configuration, because the GD structure supports SEWs with longer propagation lengths at a lower frequency interval, the GD structure can have a higher SEW thermal conductivity. However, when it is applied to other materials, it should be noted that depending on the conditions where $\epsilon_{d}^{2} \epsilon_{s}-\left(\epsilon_{d}-\epsilon_{s}\right)\left|\epsilon_{g}\right|^{2}>0$ hold, either the GD or the DG structure can be beneficial in carrying thermal energy.

\section{Glass-dielectric-glass structures}

For the three-layer structure shown in Fig. 1(c), the following dispersion relation can be derived from the Maxwell equation with proper boundary conditions [26,28]:

$$
\tanh \left(p_{d} d_{d}\right)=-\frac{\alpha_{d g}\left[1+\alpha_{s g} \tanh \left(p_{g} \frac{d_{g}}{2}\right)\right]\left[\alpha_{0 g}+\tanh \left(p_{g} \frac{d_{g}}{2}\right)\right]+\alpha_{d g}\left[\alpha_{s g}+\tanh \left(p_{g} \frac{d_{g}}{2}\right)\right]\left[1+\alpha_{0 g} \tanh \left(p_{g} \frac{d_{g}}{2}\right)\right]}{\alpha_{d g}^{2}\left[1+\alpha_{s g} \tanh \left(p_{g} \frac{d_{g}}{2}\right)\right]\left[1+\alpha_{0 g} \tanh \left(p_{g} \frac{d_{g}}{2}\right)\right]+\left[\alpha_{s g}+\tanh \left(p_{g} \frac{d_{g}}{2}\right)\right]\left[\alpha_{0 g}+\tanh \left(p_{g} \frac{d_{g}}{2}\right)\right]}
$$

where all the parameters have been previously defined. As in previous sections, an approximate analytical solution for $\beta_{I}$ of SEWs supported in a glass-dielectric-glass (GDG) structure can be expressed as follows:

$$
\beta_{I, \mathrm{GDG}} \approx \frac{D_{d, I} d_{g} \epsilon_{g, I} \epsilon_{s}^{1.5}\left(A_{I}^{\prime} D_{d, I} \epsilon_{0}-D_{0} \epsilon_{d}\right) B_{\mathrm{GDG}} k_{0}^{2}}{2 \epsilon_{d}^{3}\left(D_{d, I} \epsilon_{0}+A_{I}^{\prime} D_{0} \epsilon_{d}\right)^{3}\left(\epsilon_{g, R}^{2}+\epsilon_{g, I}^{2}\right)}
$$

where

$$
\begin{aligned}
B_{\mathrm{GDG}}= & 2 \epsilon_{d}^{2}\left(\epsilon_{d}-\epsilon_{s}\right)\left[\epsilon_{0}^{2} \epsilon_{s}+\left(\epsilon_{g, R}^{2}+\epsilon_{g, I}^{2}\right)\left(-\epsilon_{0}+\epsilon_{s}\right)\right] \\
& +A_{I}^{\prime 2}\left(\epsilon_{0}-\epsilon_{d}\right)\left[\left(\epsilon_{g, R}^{2}+\epsilon_{g, I}^{2}\right)\left(\epsilon_{d}-\epsilon_{s}\right)+\epsilon_{d}^{2} \epsilon_{s}\right] \\
& \times\left[\epsilon_{0} \epsilon_{d}-\left(\epsilon_{0}+\epsilon_{d}\right) \epsilon_{s}\right]+2 A_{I}^{\prime} D_{0} D_{d, I} \epsilon_{0} \epsilon_{d} \\
& \times\left[\epsilon_{d}^{2} \epsilon_{s}-\left(\epsilon_{d}-\epsilon_{s}\right)\left(\epsilon_{g, R}^{2}+\epsilon_{g, I}^{2}\right)\right] .
\end{aligned}
$$

In order to compare the SEW thermal conductivity of the GDG structure with those of the GD and DG structures, $\beta_{I, \mathrm{DG}}-\beta_{I, \mathrm{GDG}}$ is calculated. Interestingly, the resulting value is found to be half of the value of $\beta_{I, \mathrm{DG}}-\beta_{I, \mathrm{GD}}$ :

$$
\begin{aligned}
\beta_{I, \mathrm{DG}}-\beta_{I, \mathrm{GDG}} & =\frac{D_{d, I} d_{g} \epsilon_{g, I} \epsilon_{s}^{1.5}\left(A_{I}^{\prime} D_{d, I} \epsilon_{0}-D_{0} \epsilon_{d}\right) C k_{0}^{2}}{2 \epsilon_{d}^{3}\left(D_{d, I} \epsilon_{0}+A_{I}^{\prime} D_{0} \epsilon_{d}\right)^{3}\left(\epsilon_{g, R}^{2}+\epsilon_{g, I}^{2}\right)} \\
& =\frac{\beta_{I, \mathrm{DG}}-\beta_{I, \mathrm{GD}}}{2} .
\end{aligned}
$$

Accordingly, $\beta_{I, \mathrm{DG}}-\beta_{I, \mathrm{GDG}}=\beta_{I, \mathrm{GDG}}-\beta_{I, \mathrm{GD}}$. Furthermore, both $\beta_{I, \mathrm{DG}}-\beta_{I, \mathrm{GDG}}$ and $\beta_{I, \mathrm{GDG}}-\beta_{I, \mathrm{GD}}$ have positive values when $\epsilon_{d}^{2} \epsilon_{s}-\left(\epsilon_{d}-\epsilon_{s}\right)\left|\epsilon_{g}\right|^{2}>0$ as $\beta_{I, \mathrm{DG}}-$ $\beta_{I, \mathrm{GD}}$ does. When $\epsilon_{d}^{2} \epsilon_{s}-\left(\epsilon_{d}-\epsilon_{s}\right)\left|\epsilon_{g}\right|^{2}>0$, it is readily observed in Fig. 6(a) that $\Lambda_{\mathrm{GD}}>\Lambda_{\mathrm{GDG}}>\Lambda_{\mathrm{DG}}$ and vice versa. Among the three structures, the GD one shows 


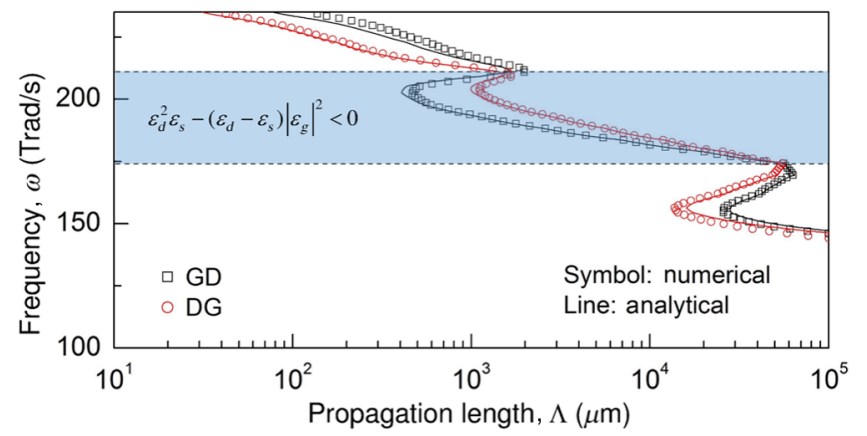

FIG. 5. The propagation lengths of the SEWs propagating along the GD and DG structures. Calculations were carried out with thicknesses of the glass and dielectric layers of $20 \mathrm{~nm}$ and $680 \mathrm{~nm}$, respectively. The dielectric function of the dielectric layer is set to 12.0 .

the highest SEW conductivity when the maximum system size is set to $10 \mathrm{~cm}$, because it supports SEWs with the longest propagation lengths in the lowest-frequency regime. Although it is not shown here, the propagation lengths of the SEWs supported in these three structures can be longer than $1 \mathrm{~m}$. This indicates that when the system size along the SEW propagation direction is smaller than the propagation length, we cannot fully exploit the benefit
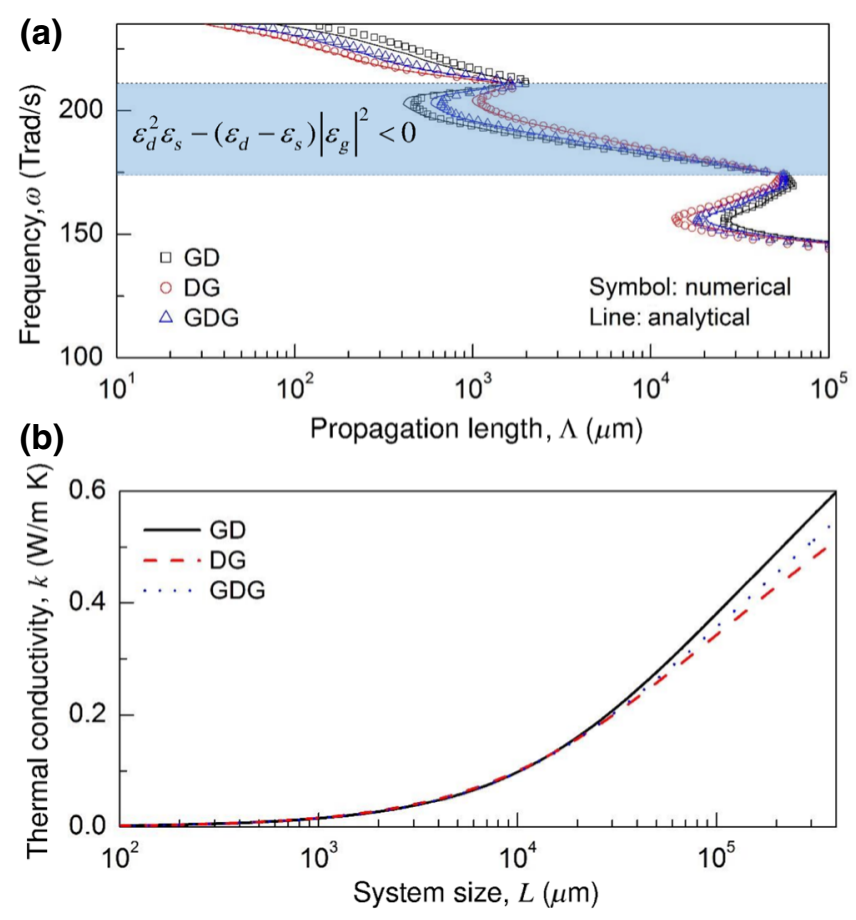

FIG. 6. (a) The propagation lengths of the SEWs supported by the GD, DG, and GDG structures. The total thickness of the glass layer is set to $20 \mathrm{~nm}$ and the thickness of the dielectric layer is $680 \mathrm{~nm}$. The dielectric function of the dielectric layer is set to 12.0. (b) The resulting thermal conductivities of each structure when $T=300 \mathrm{~K}$. of the long propagation length, but as this system size increases, its effect becomes stronger, such that the SEW thermal conductivity increases with the system size $L$, as can be seen in Fig. 6(b). Due to long propagation lengths $(\Lambda>1 \mathrm{~m})$ in the low-frequency regime, the asymptote for each configuration can be observed when the system size $L$ is larger than $10 \mathrm{~m}$, as can be inferred from the definition $1 / \Lambda_{\text {eff }}=1 / \Lambda+1 / L$.

\section{DISCUSSION}

As discussed in the previous section, $\epsilon_{d}^{2} \epsilon_{s}-\left(\epsilon_{d}-\right.$ $\left.\epsilon_{s}\right)\left|\epsilon_{g}\right|^{2}$ can act as the indicator for determining the best structure for carrying thermal energy with SEWs. The expression $\epsilon_{d}^{2} \epsilon_{s}-\left(\epsilon_{d}-\epsilon_{s}\right)\left|\epsilon_{g}\right|^{2}>0$ can be rewritten as $\left|\epsilon_{g}\right|^{2}<\left(\epsilon_{d}^{2} \epsilon_{s}\right) /\left(\epsilon_{d}-\epsilon_{s}\right)$ when $\epsilon_{d}-\epsilon_{s}>0$. Therefore, we can infer that if the glass layer is replaced by a material that has a dielectric function with a small absolute value, the propagation length for the GD structure will be the longest among the three structures; otherwise, the SEWs for the DG structure will have the longest propagation lengths. In Fig. 7, the propagation lengths for the GD, DG, and GDG structures are shown when glass layers are replaced by $\mathrm{SiC}$ and $\mathrm{MgF}_{2}$. As predicted, for $\mathrm{SiC}$, which has a higher dielectric function than glass in the lower-frequency regime, the DG structure shows the longest propagation length at the lowest frequency at which SEWs can exist. On the other hand, for $\mathrm{MgF}_{2}, \epsilon_{d}^{2} \epsilon_{s}-\left(\epsilon_{d}-\epsilon_{s}\right)\left|\epsilon_{\mathrm{MgF}_{2}}\right|^{2}>0$ holds for the entire frequency interval and, thus, $\Lambda_{\mathrm{GD}}>$ $\Lambda_{\mathrm{GDG}}>\Lambda_{\mathrm{DG}}$ can be observed for the frequency interval in which the SEWs exist. Although we have simplified the analytical solutions for various structures, these solutions can be applied to a wide range of materials and they enable us to predict the structure that holds SEWs with the longest propagation lengths.

In addition, it is important to note that calculation of the SEW thermal conductivity using the analytical solution requires a significantly reduced calculation time compared to that for the numerical solution. Accordingly, the optimal configurations for maximizing the SEW thermal conductivity can be found by employing a genetic algorithm. As in Table I, the maximum calculated SEW thermal conductivity of $0.974 \mathrm{~W} / \mathrm{m} \mathrm{K}$, which is $70 \%$ of the thermal conductivity of bulk glass, is obtained for a GD structure with $\epsilon_{s}=1.24$. For that configuration, the exact numerical solution results in a SEW thermal conductivity of $1.00 \mathrm{~W} / \mathrm{m} \mathrm{K}$. In other words, the maximum thermal conductivity can be obtained with an error less than $3 \%$ in a much shorter calculation time by employing the analytical solution. While varying the range for the dielectric function of the dielectric layer and the substrate, and the maximum system size, the error is kept to less than $5 \%$. The analytical solution can be used to conduct multiple optimization processes under various boundary conditions with high accuracy. As a result, in Table I, it is readily 

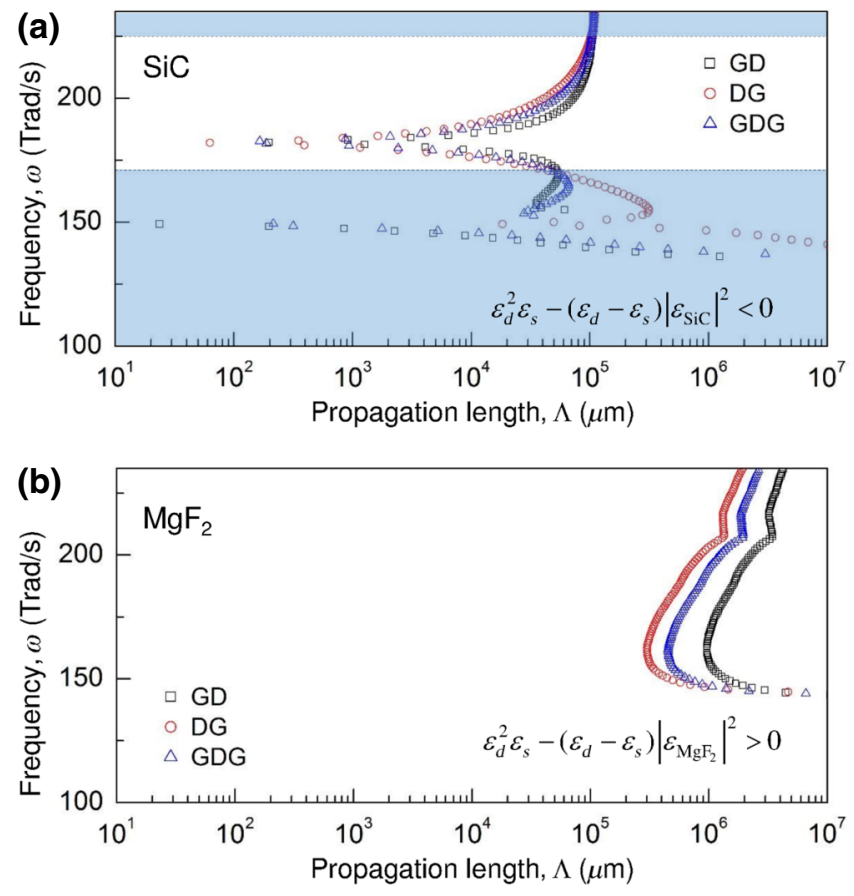

FIG. 7. The propagation lengths of the SEWs supported in the GD, DG, and GDG structures. The total thickness of the $\mathrm{SiC} / \mathrm{MgF}_{2}$ layer is $20 \mathrm{~nm}$ and the thickness of the dielectric layer is $680 \mathrm{~nm}$. The dielectric function of the dielectric layer is set to 12.0 (a) Glass is replaced by SiC. (b) Glass is replaced by $\mathrm{MgF}_{2}$.

noted that a thinner glass layer, a larger dielectric function of the dielectric layers, and a smaller dielectric function of the substrate are beneficial for enhancing SEW thermal conductivity.

It is worthwhile mentioning that the SEW thermal conductivity is a thermal-energy transport mechanism

TABLE I. Bounds for the variables and the corresponding optimal configuration and SEW thermal conductivity for the GD structure at $T=300 \mathrm{~K}$.

\begin{tabular}{|c|c|c|c|c|}
\hline Variable & $\begin{array}{l}\text { Lower } \\
\text { bound }\end{array}$ & $\begin{array}{l}\text { Upper } \\
\text { bound }\end{array}$ & $\begin{array}{c}\text { Optimal } \\
\text { configuration }\end{array}$ & $\begin{array}{l}\text { SEW thermal } \\
\text { conductivity } \\
\text { [error] } \\
(\mathrm{W} / \mathrm{m} \mathrm{K})\end{array}$ \\
\hline$d_{g}(\mathrm{~nm})$ & 20 & 1000 & 20 & \\
\hline$d_{d}(\mathrm{~nm})$ & 20 & 1000 & 695 & $0.512[1.8 \%]$ \\
\hline$\epsilon_{d}$ & 1 & 16 & 16 & \\
\hline$\epsilon_{s}$ & - & - & Set to 1.24 & \\
\hline$d_{g}(\mathrm{~nm})$ & 5 & 1000 & 5 & \\
\hline$d_{d}(\mathrm{~nm})$ & 5 & 1000 & 705 & $0.974[2.7 \%]$ \\
\hline$\epsilon_{d}$ & 1 & 16 & 16 & \\
\hline$\epsilon_{s}$ & - & - & Set to 1.24 & \\
\hline$d_{g}(\mathrm{~nm})$ & 5 & 1000 & 5 & \\
\hline$d_{d}(\mathrm{~nm})$ & 5 & 1000 & 600 & $1.27[4.5 \%]$ \\
\hline$\epsilon_{d}$ & 1 & 16 & 16 & \\
\hline$\epsilon_{s}$ & 1.1 & 16 & 1.1 & \\
\hline
\end{tabular}

additional to that of phonons. Although the phonon mean free path in an amorphous thin glass layer is very short, such that the phonon-boundary scattering does not significantly change the lattice thermal conductivity (i.e., $\left.k_{\text {lattice }} \approx 1.4 \mathrm{~W} / \mathrm{m} \mathrm{K}\right)[1,29]$, it should be noted that the maximum SEW thermal conductivity $k_{\mathrm{SEW}}$ is found to be $1.27 \mathrm{~W} / \mathrm{m} \mathrm{K}$ when the thickness of the glass layer is 5 $\mathrm{nm}$, as reported in Table I. Consequently, this additional energy transport by SEWs is a compelling tool to enhance the spreading of heat in a nanolayered system.

\section{CONCLUSIONS}

In this work, the dispersion relations of SEWs propagating along the GD, DG, and GDG structures are derived and analyzed along with their corresponding analytical solutions for the in-plane wave vector. The impact of various factors affecting the propagation length and thermal conductivity is discussed based on the analytical solutions. It is found that the frequency interval in which the SEWs exist is almost independent of the dielectric function of the glass layer. Furthermore, the SEW propagation length of each structure is compared under different conditions and the indicator for determining the structure in which SEWs with the longest propagation lengths can be supported is found. The propagation lengths of the GDG structure are found to be between those of the DG and GD structures. The optimization to obtain maximum SEW thermal conductivity is also conducted using a genetic algorithm, yielding a SEW thermal conductivity of $1.27 \mathrm{~W} / \mathrm{m} \mathrm{K}$, similar to the thermal conductivity of bulk glass. It is important to understand that thermal energy transport by SEWs has a different nature than conventional thermal conduction; that is, its relatively long propagation length indicates that the energy is weakly absorbed by the substrate. Accordingly, it is a quite effective channel via which to spread heat from hot spots. Because Si, which is the most widely used material in the semiconductor industry, has a dielectric function around 11.7 at $100 \mathrm{Trad} / \mathrm{s}$ and is almost lossless [27], the analysis conducted in this work provides guidelines for enhancing the SEW thermal conductivity of nanosized glass layers combined with $\mathrm{Si}$ ones, thereby mitigating the hot-spot issue in electronic devices.

\section{ACKNOWLEDGMENTS}

This research was supported by the Basic Science Research Program (Grants No. NRF-2017R1A2B2011192, No. NRF-2017R1A2B3009610, No. NRF-2018R1A6A3A 01012563, and No. NRF-2019R1A2C2003605) through the National Research Foundation of Korea (NRF) funded by the Ministry of Science and ICT. The stay of M. Lim at CentraleSupélec has been supported by the Erasmus Mundus EASED program (Grant No. 2012-5538/004-001) coordinated by CentraleSupélec. 
[15] M. Lim, J. Song, J. Kim, S. S. Lee, I. Lee, and B. J. Lee, Optimization of a near-field thermophotovoltaic system operating at low temperature and large vacuum gap, J. Quant. Spectrosc. Radiat. Transf. 210, 35 (2018).

[1] D.-Z. A. Chen, A. Narayanaswamy, and G. Chen, Surface phonon-polariton mediated thermal conductivity enhancement of amorphous thin films, Phys. Rev. B 72, 155435 (2005).

[2] D.-Z. A. Chen and G. Chen, Heat flow in thin films via surface phonon-polaritons, Front. Heat Mass Transf. 1, 023005 (2010).

[3] J. Ordonez-Miranda, L. Tranchant, T. Tokunaga, B. Kim, B. Palpant, Y. Chalopin, T. Antoni, and S. Volz, Anomalous thermal conductivity by surface phonon-polaritons of polar nano thin films due to their asymmetric surrounding media, J. Appl. Phys. 113, 084311 (2013).

[4] J. Ordonez-Miranda, L. Tranchant, Y. Chalopin, T. Antoni, and S. Volz, Thermal conductivity of nano-layered systems due to surface phonon-polaritons, J. Appl. Phys. 115, 054311 (2014).

[5] J. Ordonez-Miranda, L. Tranchant, B. Kim, Y. Chalopin, T. Antoni, and S. Volz, Quantized Thermal Conductance of Nanowires at Room Temperature due to Zenneck Surface-phonon Polaritons, Phys. Rev. Lett. 112, 055901 (2014).

[6] L. Tranchant, J. Ordonez-Miranda, T. Matsumoto, S. Gluchko, T. Antoni, S. Volz, and K. Miyazaki, in 2015 21st International Workshop Thermal Investigations of ICs and Systems (THERMINIC) (IEEE, Paris, 2015).

[7] Z. M. Zhang, Nano/Microscale Heat Transfer (McGrawHill, New York, 2007).

[8] O. Semenov, A. Vassighi, and M. Sachdev, Impact of selfheating effect on long-term reliability and performance degradation in CMOS circuits, IEEE Trans. Device Mater. Rel. 6, 17 (2006).

[9] H. Raether, Surface Plasmons on Smooth and Rough Surfaces and on Gratings (Springer-Verlag, Berlin, 1988).

[10] J. J. Burke, G. I. Stegeman, and T. Tamir, Surface-polaritonlike waves guided by thin, lossy metal films, Phys. Rev. B 33, 5186 (1986).

[11] F. Yang, J. R. Sambles, and G. W. Bradberry, Long-range surface modes supported by thin films, Phys. Rev. B 44, 5855 (1991).

[12] T. Kume, T. Kitagawa, S. Hayashi, and K. Yamamoto, Long-range surface modes supported by $\mathrm{SiO}_{2}-\mathrm{Ag}$ composite thin films, Surf. Sci. 395, 23 (1998).

[13] A. Dereux, J.-P. Vigneron, P. Lambin, and A. A. Lucas, Polaritons in semiconductor multilayered materials, Phys. Rev. B 38, 5438 (1988).

[14] J. Mendialdua, A. Rodriguez, M. More, A. Akjouj, and L. Dobrzynski, Bulk and surface phonon polaritons in threelayer superlattices, Phys. Rev. B 50, 14605 (1994).
[16] P. Ben-Abdallah, K. Joulain, and A. Pryamikov, Surface Bloch waves mediated heat transfer between two photonic crystals, Appl. Phys. Lett. 96, 143117 (2010).

[17] S.-A. Biehs, M. Tschikin, R. Messina, and P. Ben-Abdallah, Super-Planckian near-field thermal emission with phononpolaritonic hyperbolic metamaterials, Appl. Phys. Lett. 102, 131106 (2013).

[18] Y. Guo, C. L. Cortes, S. Molesky, and Z. Jacob, Broadband super-Planckian thermal emission from hyperbolic metamaterials, Appl. Phys. Lett. 101, 131106 (2012).

[19] R. Wang, J. Chen, Y. Xiang, Y. Kuai, P. Wang, H. Ming, J. R. Lakowicz, and D. Zhang, Two-Dimensional Photonic Devices Based on Bloch Surface Waves with OneDimensional Grooves, Phys. Rev. Appl. 10, 024032 (2018).

[20] S. Gluchko, B. Palpant, S. Volz, R. Braive, and T. Antoni, Thermal excitation of broadband and long-range surface waves on $\mathrm{SiO}_{2}$ submicron films, Appl. Phys. Lett. 110, 263108 (2017).

[21] M. Lim, J. Song, S. S. Lee, and B. J. Lee, Tailoring near-field thermal radiation between metallo-dielectric multilayers using coupled surface plasmon polaritons, Nat. Commun. 9, 4302 (2018).

[22] B. Song, D. Thompson, A. Fiorino, Y. Ganjeh, P. Reddy, and E. Meyhofer, Radiative heat conductances between dielectric and metallic parallel plates with nanoscale gaps, Nat. Nanotechnol. 11, 509 (2016).

[23] H. Iizuka and S. Fan, Significant Enhancement of NearField Electromagnetic Heat Transfer in a Multilayer Structure Through Multiple Surface-States Coupling, Phys. Rev. Lett. 120, 063901 (2018).

[24] R. Messina and P. Ben-Abdallah, Graphene-based photovoltaic cells for near-field thermal energy conversion, Sci. Rep. 3, 1383 (2013).

[25] Y. De Wilde, F. Formanek, R. Carminati, B. Gralak, P.-A. Lemoine, K. Joulain, J.-P. Mulet, Y. Chen, and J.-J. Greffet, Thermal radiation scanning tunnelling microscopy, Nature 444, 740 (2006).

[26] C. Yeh and F. I. Shimabukuro, The Essence of Dielectric Waveguides (Springer, New York, 2008).

[27] E. D. Palik, Handbook of Optical Constants of Solids (Academic Press, San Diego, 1985), Vol. 1.

[28] C. Yeh and G. Lindgren, Computing the propagation characteristics of radially stratified fibers: An efficient method, Appl. Opt. 16, 483 (1977).

[29] S.-M. Lee and D. G. Cahill, Heat transport in thin dielectric films, J. Appl. Phys. 81, 2590 (1997). 